\section{Constrained SPSA Controller for Operations Processes}

\author{
Fahimeh Rezayat
}

\begin{abstract}
Continuous quality improvement calls for employing methodologies that assist in continual reduction of variations in process performance characteristics around their target values. In a complex operations process the underlying structure of the process is unknown to the operations managers. Hence, identification of the source of variations and variation reduction are difficult and time consuming. Under the assumption that the process design is capable of producing products that meet customer's requirements, the emphasis is on continually improving performance and conformance dimensions of the quality of a complex/ nonlinear operations process when there exists almost no knowledge about the process structure. The study considers a case in which some of the operations process parameters/ inputs are required to take values in pre- specified ranges. To improve the process performance while accounting for these requirements, the study employs a neural network-based model-free controller along with the penalty function. Simultaneous perturbation stochastic gradient approximation method is used to iteratively estimate the weights of neural network and as a result to estimate the control values. Further, the study uses a special cause control chart to monitor the performance of the controller in reducing the process variations and to signal the change in the process dynamics. Simulation findings indicate that when incorporating the prespecified requirements, directly, in estimating the neural network's weight, the neural network model-free provides control values (inputs) that result in fewer nonconforming outputs than when the requirements are not incorporated in optimization process.
\end{abstract}

\section{INTRODUCTION}

The objective of any business organization is to produce goods and services that meet customers' needs. Recently a growing emphasis is given on improving operation processes. This study stresses quality improvement for the process industries. In the process industries observations are serially correlated, which results in systematic variation in the observations. When the systematic variation in observations is large, product quality is influenced. Note that the process parameter values (input values) or the process dynamics throughout the operations process may change from the original design values. When these changes are large they cause deviations of the product attributes values from the target values (special causes variations). The goal is removing/reducing the large systematic variations and the special cause variations as much as possible.

In the past, the two general approaches in industry-statistical process control and engineering process control—were independently used in reducing variability of the key product attributes around target values. Today, there exists an on-going realization that higher quality will be gained by implementing these approaches in a complementary fashion (for example, see [1]-[5]).

Control charts of the statistical process control approach (SPC) are commonly used to monitor and verify that a process remains stable and identify the special cause variations. Traditional statistical process control approaches assume that

1) consecutive observations from a process are independent;

Manuscript received August 19, 1998; revised July 2, 1999 and July 26, 1999. This work was supported in part by the California State University, Dominguez Hills, RSCAAP Summer Fellowship. This paper was recommended by Associate Editor Y. Narahari.

The author is with California State University, Dominguez Hills, Carson, CA 90747 USA (e-mail: frezayat@ soma.csudh.edu).

Publisher Item Identifier S 1083-4427(99)08398-8.
2) under normal conditions the process variability is driven by common causes, whose effect is impossible or too expensive to reduce;

3) an increase in variability or unfavorable changes in the process mean are due to special causes which are typically related to the presence of differences among operators, tools, raw materials, or days of the week.

Once the special causes are eliminated, the residual variability of the data is due to common causes (e.g., variations in environmental conditions) and is assumed to be too expensive to control [6]. The methods that are commonly used to identify the cause (source) of the variations range from the simple Pareto analysis, cause and effect diagrams, and scatter (correlation) diagrams to the Bayesbased methods. In many practical applications the special causes of variability cannot in principle be eliminated or even reduced to such an extent that their presence could be considered a negligible factor. In industrial situations involving assignable causes of variability, when a rapid response is essential, the best that can be done is to achieve a condition in which the process can produce acceptable output despite such causes, as long as their impact is not excessively large.

Engineering process control aims to address this issue by reducing systematic variations. It compensates for the true cause of variation by manipulating some other parameters of the process and by constructing a dynamic model, feedback control, feed-forward control, or sometimes a combination of them [7]. In addition to knowledge of the essential parameters of an operations process, the engineering process control typically requires knowledge of the correct form of the functional relationship between the mean value of the key product attributes and the levels of control factors (process parameters), e.g., raw materials. For systems in which the relationships are nonstationary/nonlinear, constructing an explicit functional relationship that adequately represents the true relationship is almost impossible. This fact and the need for better control of increasingly complex dynamic systems (such as modern manufacturing systems) under significant uncertainty and nonlinearity has led to reevaluation of the conventional control methods. Neural networks appear to offer new promising ways toward solving some of the most difficult control problems (for example, see [8] and [9]).

Neural networks are a type of AI system effective for control of complex processes in which the underlying structure may change or exhibit an ill-structured nature. Spall and Cristion [10], [11] developed the simultaneous perturbation stochastic approximation (SPSA) based-neural network controller. This innovative neural network-based, model-free controller does not need the construction or assumption of a separate model (either $\mathrm{NN}$ or other parametric type such as linear or nonlinear ARMA) for the underlying process equations. It takes information about the current state of the system and produces a set of control actions to modify or improve the future state of the system. Since it is not tied to a prior model, the controller has a potential advantage in handling changes in the underlying dynamics of the process.

The NN controller is appropriate when the system can tolerate nonoptimal control (training process) and the regularity conditions in [12] are met, or when the NN controller can be trained via simulation of the process (in such cases the issue of being able to tolerate nonoptimal controls is less relevant since no physical damage will be done). Then the NN controller would be used for adjusting the operations process when the monitoring chart provides signals for 
changes in the process dynamic. SPSA uses noisy measurements of the objective function and does not require direct gradient or higher derivative computation. The main input at each iteration of SPSA is an approximation to the gradient vector that is based on two measurements of the objective function, independent of the problem dimension $p$ (i.e., the number of the NN weight parameters).

The fact that in industrial practice (in continuous as well as discrete production processes) observations that are actually serially correlated implies that traditional statistical process control charts may be ineffective for monitoring process quality (for example, see [1], [3], or [13]). Control charts such as cumulative sum (CUSUM) control chart [13], the exponentially weighted moving average (EWMA) control chart [2], and special cause control (SCC) chart [14] are proposed for monitoring autocorrelated observations. Rezayat [5] showed through a simulation study, that for the dynamic process under study the SCC outperformed EWMA and CUSUM control charts in identifying the change in the process dynamics (mean shift and change to the process dynamics).

The work reported here is built on prior studies in [4] and [5]. It employs simulation to evaluate the performance of the SPSAbased NN controller in reducing the variations in process performance characteristics around their target values when penalty function is used to incorporate the constraints on input and output values of the operations into the optimization process. For ease of communication from here on, we call it "constrained NN controller." To evaluate the performance of the constrained $\mathrm{NN}$ controller we compare the findings of the simulation study with that of SPSA-based NN controller without including the constraints, which we call "unconstrained NN controller."

The following section provides a brief review of SPSA-based neural network controller and its extension for the case of the constrained optimization. Section III presents the simulation results and conclusions.

\section{Methodology}

\section{A. A Brief Review of the SPSA-Based NN Controller}

Consider an operations process whose output vector at time $k+1$ is given by

$$
x_{k+1}=\phi_{k}\left(x_{k}, u_{k}, w_{k}\right)
$$

where

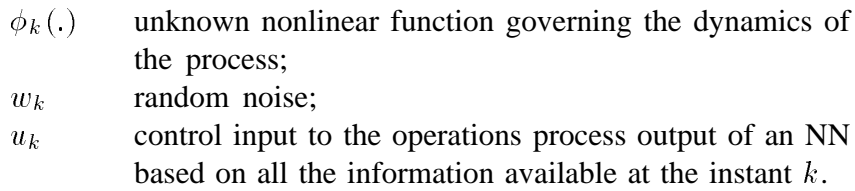

Hence, there will be a vector of connection weights $\theta_{k}$ associated to the NN producing $u_{k}$ that must be estimated. The goal is to estimate connection weights $\theta_{k}$ in a manner that results in a sequence of control values $\left\{u_{k}\right\}$ that drive the process output $\left(x_{k+1}\right)$ close to the target value $\left(t_{k+1}\right)$. In other words, the goal is to find a sequence of $\theta_{k}$ that minimizes some loss function $L_{k}\left(\theta_{k}\right)$. A common loss function is the one-step-ahead quadratic loss

$$
L_{k}\left(\theta_{k}\right)=E\left[\left(x_{k+1}-t_{k+1}\right)^{T} A_{k}\left(x_{k+1}-t_{k+1}\right)+u_{k}^{T} B_{k} u_{k}\right],
$$

where the $A_{k}$, and $B_{k}$ are positive semi-definite matrices reflecting the relative weight to put on deviations from the target and on the cost associated with larger values of $u_{k}$. A special case of the loss function is when $B_{k}=0$, and $A_{k}=I$, where the optimal controller value minimizes the square of deviation from the target value.
For estimating the $\mathrm{NN}$ weights, existing literature starts with initial guess values for the $\mathrm{NN}$ weights $\left(\theta_{0}\right)$ and recursively estimates the $\mathrm{NN}$ weights $\left(\theta_{k}\right)$ using a stochastic approximation algorithm of the form

$$
\hat{\theta}_{k}=\hat{\theta}_{k-1}-a_{k} \hat{g}_{k}\left(\hat{\theta}_{k-1}\right)
$$

where

$\hat{\theta}_{k} \quad$ estimate of $\theta_{k}$ at the $k$ th iteration;

$a_{k} \quad$ scalar gain sequence satisfying certain conditions;

$\hat{g}_{k}($.$) estimate or observation of the gradient of a loss function$ $\left(L_{k}\right)$.

Traditional NN controllers are designed using standard gradient-based search techniques to estimate $\mathrm{NN}$ weights (e.g., [15]), and the value of $\hat{g}_{k}($.$) requires knowledge of the process dynamics (say, as in$ back-propagation). When the process dynamics are unknown, the standard gradient-based search techniques can not be used. Spall and Cristion [10], [11] proposed using the "simultaneous perturbation" approximation of gradient [12] in estimating $\mathrm{NN}$ weights, where the gradient estimate, $\hat{g}_{k}($.$) , at each k$, is based on two instantaneous observed values (or noisy observed values) of loss function $L_{k}$, say $\hat{L}_{k}^{( \pm)}$. For more on the SPSA approximation, see [11] and [16]. Equation (2.4) shows the calculation of the $h$ th component of the gradient estimate, $\hat{g}_{k h}(),. h=1,2, \cdots, p$, via SPSA

$$
\hat{g}_{k h}\left(\hat{\theta}_{k-1}\right)=\left(\hat{L}_{k}^{(+)}-\hat{L}_{k}^{(-)}\right) / 2 c_{k} \Delta_{k h} \quad h=1,2, \cdots, p
$$

where $p$ represents the number of connection weights of the $\mathrm{NN}$, $\hat{L}_{k}^{( \pm)}$are estimated values of $L_{k}=L\left(\hat{\theta}_{k} \pm_{c k} \Delta_{k}\right)$ using the observed, $x_{k+1}^{( \pm)}, u_{k}^{( \pm)}$and $t_{k+1}$ (note that all $p$ elements of the gradient vector use the same two loss values), and $u_{k}^{( \pm)}$are control values and they are the NN output. Their values are based on the NN $p$ element weight vector $\theta_{k}=\hat{\theta}_{k-1} \pm c_{k} \Delta_{k}$, where $\Delta_{k}=\left\{\Delta_{k h}\right\}$ is a vector of $p$ symmetrically distributed (about 0 ) i.i.d. random variables with $E\left(\Delta_{k h}^{-2}\right)$ uniformly bounded. Note that $\Delta_{k h}$ may not be either uniformly or normally distributed (it can be symmetrically Bernoulli \pm 1 distributed which we will use in this study); $x_{k+1}^{( \pm)}$are process outputs based on $u_{k}^{( \pm)} ; t_{k+1}$ are desired target values for $x_{k+1}^{( \pm)} ;\left\{a_{k}\right\}$ and $\left\{c_{k}\right\}$ are a sequence of positive numbers. If the operations dynamics and loss function are constant, typically, $c_{k}$, and $a_{k}$ should satisfy standard conditions, e.g., $a_{k} \rightarrow 0, c_{k} \rightarrow 0$, $\Sigma_{k=0}^{\infty}\left(a_{k} / c_{k}\right)<\infty$; if the operations dynamics are changing, picking constant coefficients $c_{k}=c$ or $a_{k}=a \forall k$ is recommended.

The essential feature of SPSA is that it requires only two noisy measurements of the objective function in approximating the gradient regardless of the dimension of the optimization problem. SPSA provides the same level of accuracy in estimating the $\mathrm{NN}$ weights with orders of magnitude fewer system measurements than the standard finite difference based approach (see [12] and [17]).

\section{B. Constrained SPSA-Based NN Controller}

In practice when solving a control or optimization problem, a solution that simultaneously satisfies a set of physical, environmental, and/or economical constraints has to be considered. For example, control variables should not assume values out of a specific range due to reasons such as safety. Also output's characteristics of an operations process must meet the customers requirements. Most often these constraints/requirements are directly incorporated in control/optimization procedure.

There are several methods for incorporating constraints into a deterministic optimization problem, e.g., the penalty function method, the barrier method, and the Lagrange Multipliers method. All these methods have stochastic analogues. When the objective function and 
constraints are only observable with some random noise, Pflug [18] using the penalty function method showed that the approximating sequences converge (point-wise) to the minimal solution set. The penalty function method is an iterative optimization method, which degrades the objective function for any violation of the constraints. The penalty function method is less restrictive than any other methods, for example, the Fiacco-McCromick (barrier) method (e.g., the measurements may fall outside the constraints but they are unacceptable).

In brief, consider a constrained optimization problem of the general form

$$
\begin{array}{ll}
\text { Minimize } & f(x), \\
\text { subject to } & h_{i}(x) \leq 0, \quad \text { for } i=1, \cdots, m .
\end{array}
$$

The penalty function $\alpha($.$) of the form$

$$
\alpha_{i}(x)=\left[\operatorname{maximum}\left\{0, h_{i}(x)\right\}\right]^{q} \quad \text { for } i=1, \cdots, m
$$

is commonly used to transform the problem to an unconstrained problem of the form

$$
\text { Minimize }\left\{f(x)+\Sigma^{m} \beta_{i}\left[\operatorname{maximum}\left\{0, h_{i}(x)\right\}\right]^{q}\right\}
$$

where $\beta_{i}$ is a penalty parameter. If $h_{i}(x) \leq 0$, then maximum $\left[0, h_{i}(x)\right]=0$; on the other hand, if $h_{i}(x)>0$, then maximum $\left[0, h_{i}(x)\right]>0$, and the penalty term $\beta_{i} h_{i}(x)^{q}$ is added for each $i$ in the objective function. Most algorithms using the penalty function employ a sequence of increasing penalty parameters. With each new value of the penalty parameter, iteration starting with the optimal solution corresponding to the previously chosen parameter value is performed. The process stops when $\beta_{i} \alpha_{i}(x)<\epsilon_{i}$ for all $i$, where $\epsilon_{i}$ is a predetermined termination scalar. For more on the penalty method, see [19] or others.

In [20], the nearest point on a parameter allowable set approach is employed to incorporate the constraints on the service time in a queuing problem when using SPSA to approximate gradients and when $\hat{\theta}_{k}=\hat{\theta}_{k-1} \pm c_{k} \Delta_{k}$ lies outside the constraint set. When the measurement outside a constraint set is not allowed in [21], it is suggested further projecting $\hat{\theta}_{k}$ onto a closed subset of the constraint set to obtain a new estimated value, $P_{k}\left(\hat{\theta}_{k}\right)$, and then applying the SPSA technique to the gradient.

Note that

1) stochastic approximation procedure which Pflug [18] employed is a modified Kiefer-Wolfowitz procedure;

2) SPSA is also a modified version of Kiefer-Wolfowitz procedure;

3) all required conditions in [12] along with the condition " $\Sigma a_{k} c_{k} \beta_{k}<\infty$ " are conditions that Pflug required for the convergence of the parameter values into the minimal set.

Hence one may use the penalty function along with SPSA to include the constraints in the optimization process.

We consider a case in which noisy information on the objective function is available and the constraints/requirements are expressed in inequality form. To account for the output constraints, we use the penalty function method at the gradient approximation step. In order to incorporate the system's input constraint, we follow [20]. We assume that our goal is to find a controller that minimizes the onestep-ahead loss function $\left(L_{k}\right)$, where the loss function is a function of output value $\left(x_{k+1}\right)$, target value $\left(t_{k+1}\right)$, and controller value $\left(u_{k}\right)$, and it is given by

$$
\operatorname{Min} E\left(\left\|x_{k+1}-t_{k+1}\right\|^{2} \mid u_{k}\right)
$$

and the constraints are

$$
\begin{array}{cc}
C 1: & E\left(\left\|x_{k+1}-t_{k+1}\right\|\right) \leq r \sigma \\
C 2: & u_{k} \epsilon S_{k}
\end{array}
$$

where $x_{k+1}=\Phi_{k}\left(x_{k}, x_{k-1}, \cdots, x_{k-s}, u_{k}, w_{k}\right), s \geq 0 ; \Phi_{k}($.$) is$ unknown, $r$ is a constant, $w_{k}$ is the noise of the dynamic process, and $\sigma$ is the standard deviation of the process noise. The goal is to choose a sequence of control vectors in $S_{k}=\left\{u_{k}:\left|u_{i k}\right| \leq\right.$ $\left.u_{i k}^{*}, i=1,2, \cdots, n\right\}$, (where $n$ is the dimension of control vectors) such that the system output $\left\{x_{k+1}\right\}$ is close to the sequence of target vectors $\left\{t_{k+1}\right\}$, where close is relative to the magnitude of the noise $\left\{w_{k}\right\}$ and the cost associated with the control. For incorporating the output constraint $(C 1)$, in the gradient approximation procedure, when calculating $\hat{L}_{k}^{( \pm)}$at each $k$, a penalty term resulting from the multiplication of a penalty parameter $\beta_{k}$ and $\max \left\{0,\left[x_{k+1}^{( \pm)}-t_{k+1}\right]^{2}\right\}$ is added to the loss function. $\beta_{k}$ is an increasing function of the deviation of the output attribute from the target value at time $k$ ( $\beta_{k}$ assumes a large penalty value when deviation is large and a smaller value when the deviation is small). To account for the input constraint $(C 2)$, whenever the NN provides an $u_{k} \notin S_{k}$, the $u_{k}$ value will be adjusted to the nearest value in the set $\left(S_{k}\right)$; otherwise the output of the NN will be used as input to the system.

\section{Simulation ExPeriment}

In this study, the batch polymerization example in [1] is employed, and it is assumed that the stochastic model constructed in [1] represents the true dynamics of the polymerization process, but that the knowledge about the process dynamics does not exist. Then the SPSA-based NN controller is used to simulate control values that reduce the polymer viscosity variations about the target value. The SCC chart is employed for monitoring the viscosity variations. The study reports the relative performance of the NN controller in maintaining the viscosity variations in the tolerance range for constrained optimization and unconstrained optimization cases, under two different experiment assumptions. In the first experiment the mean value of the process is shifted, while in the second experiment the dynamics of the process is changed to nonlinear. Assumption for both experiments is that the NN controller has no knowledge about the changes. Therefore, the time the controller learns about the change (and SCC signals it) and the number of the times the viscosity variations fall outside of SCC chart (number of nonconforming outputs) are used as criteria for evaluating controller's performance. The findings of the simulation study in Section III-A indicates that including constraints via penalty function in the gradient approximation procedure has improved the learning rate of the NN controller and has resulted in fewer nonconforming outputs than for the unconstrained method.

\section{A. Simulation Study}

For the batch polymerization example in [1], it is assumed that each batch cycle consists of the following steps: charge the reactor, run the reaction, empty the reactor contents into a holding tank for subsequent processing, engage cleaning procedures, and recharge to begin the next batch. Intrinsic viscosity, a key quality characteristic of the polymer, is measured at the completion of each batch. Turnaround time is such that the viscosity measurement from the most recent batch produced in a given reactor is available when the reactor is prepared for a new batch. The statistically significant viscosity variations about a target level of 100 viscosity units are observed. In analyzing the cause of viscosity variations, Vander Wiel et al. [1] reported that autocorrelation among viscosity measurements exist. Autocorrelations stem from several factors. For example, mechanical 
considerations prevent a reactor from being fully emptied between batches. They also reported that the level of catalyst has the highest effect on the level of viscosity and must be adjusted in order to reduce the viscosity deviations from the target value. Based on the observed intrinsic viscosity deviations from its target value and the catalyst deviations from its nominal value, Vander Wiel et al. [1] provided the following empirical model for the batch $k$ :

$$
x_{k}=0.8 x_{k-1}+1.5 u_{k-1}-1.2 u_{k-2}+w_{k}-0.22 w_{k-1}
$$

where $x_{k}$ is observed viscosity deviation from a 100 unit target viscosity, $u_{k-1}$ is catalyst deviation from nominal (50 units), and $w_{k}$ is independent and normally distributed $\left(N\left(0, \sigma_{w}^{2}\right)\right.$, where $\sigma_{w}=$ 2.798). Equation (3.1) employs the compromise parameter estimate values of the Vander Wiel et al. [1] empirical study. Under the assumption that the most recent viscosity measurement is available, and based on the minimum mean square errors, Vander Wiel et al. [1] reported the following so-called pure-one-step adjustment rule

$$
u_{k}=0.8 u_{k-2}-0.4 x_{k-1} \text {. }
$$

This simulation study employs the SPSA-based NN controller discussed in Section II instead of algorithm (3.2). The data are generated according to the empirical model (3.1) and the controller here assumes no knowledge of (3.1). The SPSA-based NN controller adjusts the level of catalyst for $i$ th batch of a reactor based on the viscosity deviation of $(i-1)$ th batch from the target value, and the catalyst deviation of $(i-2)$ th batch from the nominal value.

The SCC chart with control limits of $\pm 3 \sigma_{w}$ is used for monitoring the viscosity variations. When the constrained optimization method is used

1) maximum allowable deviation of the catalyst from its nominal value is subjectively set to 7 and when the deviation in catalyst from its nominal value falls out of the allowable range of $[-7$, 7], its value is adjusted to -7 or 7 whichever is the nearest;

2) penalty factor, $\beta_{k}$, assumes the following arbitrarily selected values: 0.001 when $1.3 \leq\left|x_{k}-t_{k}\right|<2.8 ; 0.01$ when $2.8 \leq\left|x_{k}-t_{k}\right|<5.8 ; 0.1$ when $5.8 \leq\left|x_{k}-t_{k}\right|<8.34$ and a value between 1 and 2.8 when $\left|x_{k}-t_{k}\right| \geq 8.34$.

Since the goal is to have zero deviation from the target value, following Taguchi [22], we have considered penalty for deviations less than $\left|3 \sigma_{w}\right|$.

The NN controller has no knowledge about the dynamics of the process and it learns about the process dynamics through the first iterations. For each case, this study conducts ten independent simulation runs, each of 1000 iterations. Each simulation run represents the performance of one reactor. The result of one iteration of a simulation run represents the polymer viscosity deviation of one production batch from the target value. The first 500 iterations of each run are used for training the $\mathrm{NN}$, the second 500 iterations are used for studying the performance of the NN. For every simulated reactor and every sequence of 100 simulated batches, the study calculates the number of simulated production batches whose viscosity deviations from the target value fall outside the SCC control chart limits.

Since the learning rate of NN has been reported in [4], the summary of the performance of the $\mathrm{NN}$ when either the mean of the process is shifted or the structure of the process has changed is reported.

First, following [1] beginning with the 584th iteration, the process mean shifted by an amount of 10.9. and the controller has to learn the change and readjust the nominal catalyst. The controller performance is reported based on the last 500 iterations of the ten simulation runs for two different experiments (constrained and unconstrained). The SCC chart, on the average, provided signals for change (shift) on the 84.6th batch when the SPSA-based NN controller is used and on the 86.1th batch when the constrained $\mathrm{NN}$ controller is used.
On the average, the unconstrained optimization method resulted in $10.2 \%$ out-of-control simulated batches between the 100th and 200th iterations, $7.1 \%$ out-of-control batches between the 200th and 300th, and $6.4 \%$ out-of-control between the 400th and 500th iterations; whereas the constrained optimization resulted in $10.4 \%$ out-of-control simulated batches between the 100th and 200th iterations, 3.6\% outof-control batches between the 200th and 300th iterations, and $1.86 \%$ between 400th and 500th iterations.

Next, at the 84th period of the last 500 iterations, the system was changed to a nonlinear system (instead of just shifting the mean). In particular, the structure is changed to

$$
\begin{aligned}
x_{k}= & 0.8 x_{k-1}+0.25 u_{k-1} x_{k-1}+1.5 u_{k-1}-1.2 u_{k-2} \\
& +w_{k}-0.22 w_{k-1} .
\end{aligned}
$$

This assumption represents the fact that there is a possibility of a change in the underlying nature of the polymerization process, for example, due to change in raw materials. Again, the controller has to learn the change and readjust the nominal catalyst. The findings indicate that, for both cases, the SCC chart provided signals almost at the same time (at the 95th simulated batch for unconstrained and at the 96th simulated batch for the constrained). On the average, the unconstrained optimization method resulted in $6.86 \%$ out-of-control simulated batches between the 100th and 200th iterations, $2 \%$ out-ofcontrol batches between the 200th and 300th iterations, and $1.86 \%$ out-of-control between the 400th and 500th iterations; whereas the constrained optimization resulted in $3.3 \%$ out-of-control simulated batches between the 100th and 200th iterations, $1.7 \%$ out-of-control batches between the 200th and 300th iterations, and $1.57 \%$ between 400th and 500th iterations.

On the whole, the findings of the study indicate that the $\mathrm{NN}$ controller is able to recognize the shift in the mean of the process more readily than the shift in the complexity of the process, and that the constrained optimization when coupled with the SPSAbased NN controller performs slightly better than the unconstrained optimization, since on average, it produces a smaller number of out-of-control simulated batches for both cases (shift in mean and structure change).

\section{REFERENCES}

[1] S. A. Vander Wiel, W. T. Tucker, F. W. Faltin, and N. Doganaksoy, "Algorithmic statistical process control: Concepts and an application," Technometrics, vol. 34, pp. 286-297, 1992.

[2] E. Yashchin, "Statistical control schemes: Methods, applications and generalizations," Int. Stat. Rev., vol. 61, pp. 41-66, 1993.

[3] D. G. Wardell, H. Moskowitz, and R. D. Plante, "Run-length distributions of special cause charts for correlated processes," (along with discussions and rejoinder), Technometrics, vol. 36, pp. 1-27, 1994.

[4] F. Rezayat, "On integration of statistical process control and engineering process control-A neural network application," in Manufacturing Decision Support Systems, Parsaei et al., Eds. London, U.K.: Chapman \& Hall, 1997, pp. 238-252

[5] _ , "On the use of SPSA-based model-free controller in quality improvement," Automatica, vol. 31, pp. 913-915, 1995.

[6] E. Yashchin, "Monitoring variance components," Technometrics, vol. 36, pp. 379-393, 1994.

[7] G. Box and A. Luceno, "Selection of sampling internal and action limit for discrete feedback adjustment," Technometrics, vol. 36, pp. 369-378, 1994.

[8] M. Agarwal, "A systematic classification of neural network-based control," IEEE Contr. Syst. Mag., pp. 75-93, Apr. 1997.

[9] M. M. Polycarpou, "Stable adaptive neural control scheme for nonlinear systems," IEEE Trans. Automat. Contr., vol. 41, pp. 447-451, Mar. 1996.

[10] J. C. Spall and J. A. Cristion, "A neural network controller for systems with unmodeled dynamics with applications to wastewater treatment," IEEE Trans. Syst., Man, Cybern. B, vol. 27, pp. 369-373, June 1997.

[11] _ , "Model free control of nonlinear stochastic systems with discrete-time measurements," IEEE Trans. Automat. Contr., vol. 43, pp. 1198-1210, 1998. 
[12] J. C. Spall, "Multivariate stochastic approximation using a simultaneous perturbation gradient approximation," IEEE Trans. Automat. Contr., vol. 37, pp. 332-341, 1992.

[13] D. C. Montgomery, "The use of statistical process control and design of experiments in product and process improvement," Trans. IIE, pp. 4-17, Nov. 1992

[14] L. C. Alwan and H. V. Roberts, "Time series modeling for statistical process control,” J. Bus. Econ. Stat., vol. 6, pp. 87-95, 1988.

[15] K. S. Narendra and K. Parthasarathy, "Identification and control of dynamical systems using neural network," IEEE Trans. Neural Networks, vol. 1, pp. 4-27, 1990.

[16] J. C. Spall, "Implementation of the simultaneous perturbation algorithm for stochastic optimization," IEEE Trans. Aerosp. Elect. Syst., vol. 34, pp. 817-823, 1998

[17] D. Chin, "Comparative study of stochastic algorithms for system optimization based on gradient approximations," IEEE Trans. Syst., Man, Cybern. B, vol. 27, pp. 244-249, Apr. 1997.

[18] G. C. Pflug, "On the convergence of a penalty-type stochastic optimization procedure," J. Inf. Optim. Sci., vol. 2, no. 3, pp. 249-258, 1981.

[19] G. P. McCormick, Nonlinear Programming. New York: Wiley, 1983.

[20] M. C. Fu and S. D. Hill, "Optimization of district event systems via simultaneous perturbation stochastic approximation," Trans. IIE, vol. 29, pp. 233-243, 1997.

[21] P. Sadegh, "Constrained optimization via stochastic approximation with a simultaneous perturbation gradient approximation," Automatica, vol. 33, pp. 889-892, 1997.

[22] Taguchi and Wu, Introduction to Off-Line Quality Control. Nagoya, Japan: Central Japan Quality Contr. Assoc., 1985. 